\title{
Profiling grapevine trunk pathogens in planta: a case for community-targeted DNA metabarcoding
}

Abraham Morales-Cruz¹, Rosa Figueroa-Balderas', Jadran F. García', Eric Tran', Philippe E. Rolshausen², Kendra Baumgartner ${ }^{3}$ and Dario Cantu ${ }^{1 *}$ (D)

\begin{abstract}
Background: DNA metabarcoding, commonly used in exploratory microbial ecology studies, is a promising method for the simultaneous in planta-detection of multiple pathogens associated with disease complexes, such as the grapevine trunk diseases. Profiling of pathogen communities associated with grapevine trunk diseases is particularly challenging, due to the presence within an individual wood lesion of multiple co-infecting trunk pathogens and other wood-colonizing fungi, which span a broad range of taxa in the fungal kingdom. As such, we designed metabarcoding primers, using as template the ribosomal internal transcribed spacer of grapevine trunk-associated ascomycete fungi (GTAA) and compared them to two universal primer widely used in microbial ecology.
\end{abstract}

Results: We first performed in silico simulations and then tested the primers by high-throughput amplicon sequencing of (i) multiple combinations of mock communities, (ii) time-course experiments with controlled inoculations, and (iii) diseased field samples from vineyards under natural levels of infection. All analyses showed that GTAA had greater affinity and sensitivity, compared to those of the universal primers. Importantly, with GTAA, profiling of mock communities and comparisons with shotgun-sequencing metagenomics of field samples gave an accurate representation of genera of important trunk pathogens, namely Phaeomoniella, Phaeoacremonium, and Eutypa, the abundances of which were over- or under-estimated with universal primers.

Conclusions: Overall, our findings not only demonstrate that DNA metabarcoding gives qualitatively and quantitatively accurate results when applied to grapevine trunk diseases, but also that primer customization and testing are crucial to ensure the validity of DNA metabarcoding results.

Keywords: High-throughput DNA sequencing, Amplicon sequencing, Metagenomics, Grapevine trunk diseases, Esca, Eutypa dieback, Botryosphaeria dieback, Phomopsis dieback

\section{Background}

Grapevine trunk diseases affect the longevity and productivity of grapevines (Vitis vinifera) in all major growing regions of the world [1-4]. They are caused by numerous species of fungi that infect and damage the wood, causing chronic infections [5-7]. Among the most common grapevine trunk diseases are Eutypa dieback (primarily caused by Eutypa lata), Esca (primarily caused by Phaeoacremonium minimum, Phaeomoniella chlamydospora, and

\footnotetext{
* Correspondence: dacantu@ucdavis.edu

1 Department of Viticulture and Enology, University of California Davis, One

Shields Ave, Davis, CA 95616, USA

Full list of author information is available at the end of the article
}

Fomitiporia spp.), Botryosphaeria dieback (primarily caused by Neofusicoccum parvum, Diplodia seriata, among other fungi in the Botryosphaeriaceae family), Phomopsis dieback (primarily caused by Diaporthe ampelina), and Black foot (caused by Cylindrocarpon, Campylocarpon, and Ilyonectria spp.) [4, 8-11]. Because of the characteristic mixed infections, trunk diseases represent a disease complex [12,13]. In addition to infections of pruning wounds by airborne and splash-dispersed spores, trunk pathogens may be introduced to a healthy vineyard by asymptomatic propagation material. Fungi associated with grapevine trunk diseases have been found in rootstock mother-plants, rooted rootstock cuttings, 
bench-grafts, and young grafted vines [14-16]. The presence of multiple species in the same vine complicates disease diagnosis and, consequently, proper timing of practices to limit infection in the vineyard and to propagate clean nursery stock.

Taxonomic identification of fungi associated with grapevine wood is currently done by the following steps: (i) plating grapevine woody tissue on nutrient-rich agar plates, (ii) hyphal-tip colony isolation to pure cultures, (iii) DNA extraction from fungal mycelium, (iv) PCR amplification of taxonomically informative loci, such as the nuclear ribosomal internal transcribed spacer (ITS), elongation factor, and $\beta$-tubulin, and (v) comparisons of amplicon sequences with sequence databases [17-19]. PCR-based diagnostics represent a significant improvement compared to traditional approaches that depend on morphological features for species identification and, thus, require skilled expertise in mycology [20]. However, these approaches still require an initial culturing step, which may limit the detection of slow-growing fungi. Alternatively, with species or genus-specific markers, PCR could be used to determine in planta the presence of certain species, thereby skipping the culturing step $[21,22]$. One limitation of this approach, however, is that it may not detect all trunk pathogens in a given sample [23, 24]. Indeed, certain combinations of fungi may be important in the severity of symptom expression [25].

Because trunk pathogens cause mixed infections, attempts have been made to characterize the composition of the trunk-pathogen community. For example, finger-printing techniques like Automated Ribosomal Intergenic Spacer Analysis (ARISA) [26] and Single-Strand Conformation Polymorphism (SSCP) $[27,28]$ have been used to compare fungal communities among different samples of grapevine wood, although these do not identify trunk pathogens to the species level.

Quantitative PCR (qPCR) has also been applied to the detection of grapevine trunk pathogens, including strategies that allow the monitoring of multiple species simultaneously [21, 22, 24, 29, 30]. A DNA macroarray system, based on reverse dot-blot hybridization containing oligonucleotides complementary to portions of the $\beta$-tubulin locus, was developed for species-level identification, specifically for detection of trunk pathogens that cause Young vine decline [23]. We previously described a strategy, based on untargeted shotgun sequencing of metagenomic DNA and RNA, to detect and quantify trunk pathogens in planta simultaneously [13]. Despite clear advantages over other approaches, this method still has its limitations, such as relying on assembled genomes, as well as costly library preparation and computationally intensive analyses.
DNA metabarcoding, which has been used extensively for the analysis of microbial communities [31-35], may provide a cheaper and more scalable method for the characterization of trunk-pathogen communities. This approach has already been applied to other pathosystems to address a variety of research objectives. For example, DNA metabarcoding has been used to identify candidate pathogens [36, 37] and potential biocontrol agents [38], to profile putative plant pathogens associated with insects [39], and to diagnose quarantine pathogens as part of national plant-protection programs [40-42]. DNA metabarcoding infers taxonomic composition of complex biological samples by amplifying, sequencing, and analyzing target genomic regions [43, 44]. The ribosomal ITS, which is under low evolutionary pressure and, thus, presents high levels of variation between closely related species, has been commonly used as a barcode for the analysis of fungal biodiversity $[45,46]$. ITS is typically amplified by universal primers that anneal to the conserved flanking sequences. The "universality" of the primers, which derives from their ability to amplify a broad range of taxonomically unrelated species across the fungal Kingdom [47], is exploited in studies that aim to profile fungal communities, typically in exploratory analyses of environmental samples. We hypothesized that although universal primers may capture broad biodiversity in exploratory analyses, they may provide less accurate representation of microbial pathogen communities than primers that are designed and optimized to amplify species known to be associated with those communities, based on prior knowledge of disease etiology. After all, grapevine-trunk diseases are one of the most widely studied disease complexes in terms of species composition (Lamichane and Venturi, 2015). In this work, we designed and evaluated metabarcoding primers that were optimized to amplify the ITS regions of grapevine trunk pathogens. By a combination of in silico simulations, and analyses of 'mock' communities, samples from controlled inoculations, and samples from symptomatic vineyards, we demonstrated that communitycustomized metabarcoding provides both qualitatively and quantitatively a more accurate representation of trunk-pathogen communities than common universal primers.

\section{Results}

\section{Primer design, selection, and validation with target species}

We designed multiple degenerate primers that target the internal transcribed spacer (ITS) of grapevine trunk-associated ascomycetes (GTAA) using the TrunkDiseaseID as reference database [20]. Primer potential was determined in silico, considering the amplicon size and estimating the number of sequence hits to the database, their alignment 
mismatches, and gap scores. From a total of twenty forward and three reverse degenerate primers, primers GTAA182f and GTAA526r (GTAA, hereinafter) performed the best and were selected for further testing. While the BITS and SP primers target the ITS1 region, the selected GTAA primers target the entire ITS2 region with the forward and reverse primers aligning to the $5.8 \mathrm{~S}$ ribosomal RNA and the large subunit ribosomal ribonucleic acid (LSU), respectively (Table 1 and Fig. 1a). The primers produced amplicons of approximately $350 \mathrm{bp}$ from isolates of seven trunk pathogens, as expected based on the amplicon size predicted from the 213 ITS sequences of ascomycetes in the TrunkDiseaseID database $(301.72 \pm 7.53$ bp; Fig. 1 b). We obtained a similar amplicon size when the GTAA primers were used to amplify total DNA extracted from naturally infected grapevines with trunk-disease symptoms, including leaf symptoms of Esca or Eutypa dieback and wood cankers (Fig. 1c). Amplicon sequences matched the correct species, when aligned to our custom grapevine trunk pathogen-focused database using BLASTn, thereby confirming the ITS2 region amplified by the GTAA primers is informative for taxonomic assignments (Additional file 1). For field samples, multiple bands in Fig. 1c (e.g.: WC4) were likely caused by the size diversity among the ITS 2 regions in the taxa amplified.

GTAA primer precision, sensitivity, efficiency, and usefulness for metabarcoding of grapevine trunk pathogens were compared to those of the BITS [48] and SP primers [49]. The BITS primers are widely used for fungal metabarcoding analysis in vineyards and grape must (e.g.: [50-54]), whereas the SP primers [49] were implemented in the Earth Microbiome Project (http:// www.earthmicrobiome.org), and were used in recent microbial ecology studies [55-57] (Table 1 and Fig. 1a). Samples were from DNA extracted from potted grapevines either inoculated with $N$. parvum or from non-inoculated controls. By sampling PCR reactions every five cycles, the GTAA amplicon was visible on an agarose gel starting at 20 cycles, whereas those of SP and BITS were visible at 25 and 30 cycles, respectively (Fig. 1d). Furthermore, SP produced multiple bands, which may be due to non-specific binding and/or chimeric amplicons. Based on $\mathrm{GPCR}$ with the same

Table 1 Metabarcoding primer sequences targeting the ITS region used in this study

\begin{tabular}{llll}
\hline Primer name & Direction & Primer sequence 5' to 3' & Citation \\
\hline GTAA & Forward & AAAACTTCAACAACGGATC & this study \\
GTAA & Reverse & TYCCTACCTGATCCGAGGTC & this study \\
BITS & Forward & CTACCTGCGGARGGATCA & {$[48]$} \\
BITS & Reverse & GAGATCCRTTGYTRAAAGTT & {$[48]$} \\
SP & Forward & CTTGGTCATTTAGAGGAAGTAA & {$[49]$} \\
SP & Reverse & GCTGCGTTCTTCATCGATGC & {$[49]$} \\
\hline
\end{tabular}

samples, the average $\mathrm{Ct}$ values for GTAA were approximately nine cycles lower than those of BITS $\left(P<1.85 \mathrm{e}^{-04}\right)$ and SP $\left(P<3.50 \mathrm{e}^{-04}\right)$ (Fig. 1e). Overall, our findings suggest a higher affinity of the GTAA primers, when amplifying samples containing grapevine trunk pathogens.

\section{In silico simulation of amplification and taxonomic classification}

We then carried out an in silico simulation that compared the potential amplification bias and taxonomic usefulness of GTAA, BITS, and SP primers. We compiled a custom database of 521 full-length ITS sequences across 17 genera of fungi commonly associated with grapevine trunk diseases (Fig. 2a, Additional file 2; [20]). We included only full-length ITS sequences to be able to compare primers that amplify different regions of the ITS (Fig. 1a). In silico amplification of each sequence in the custom database was carried out considering all alternative sequences of degenerated primers and allowing a series of mismatches between primer and template sequences. In silico amplification was carried out testing all possible combinations of allowed mismatches, from 0 to 5 mismatches in the first five nucleotides in $5^{\prime}$ of the primer and 0 to 2 mismatches in the remaining $3^{\prime}$ nucleotides of the primer. GTAA primers amplified a higher number of sequences than BITS and SP primers, for every parameter tested (Fig. 2b). When no mismatches between primer and target were allowed, GTAA primers amplified $85.80 \%$, SP primers amplified $13.63 \%$, and BITS primers were predicted to amplify none of the sequences in the database. When at least two mismatches were allowed in the tail of the primer, BITS and SP primers amplified only 16.70 and $30.33 \%$ of target sequences, respectively, whereas GTAA primers amplified $86.75 \%$. With the most permissive parameters, GTAA primers amplified $98.08 \%$ of the sequences, and BITS and SP primers amplified 97.89 and $25.91 \%$, respectively. The requirement of multiple mismatches for BITS primers to achieve a similar number of sequences as GTAA primers is consistent with the cycle-sampling results (Fig. 1d), and suggests that GTAA primers are more efficient than BITS at amplifying the ITS of grapevine trunk pathogens.

To determine if amplicons generated by GTAA primers are informative for taxonomic assignment, we analyzed with Mothur [58] the amplicons that were generated by the simulation using the 521 full-length ITS sequences as database. Mothur uses a $k$-mer based approach to compare the query to the database and assigns the query to the taxonomy with the highest probability. By comparing the assigned genera (observed) with the expected genera for each primer set we assessed false positive (FP; i.e., erroneously assigned), false negative 

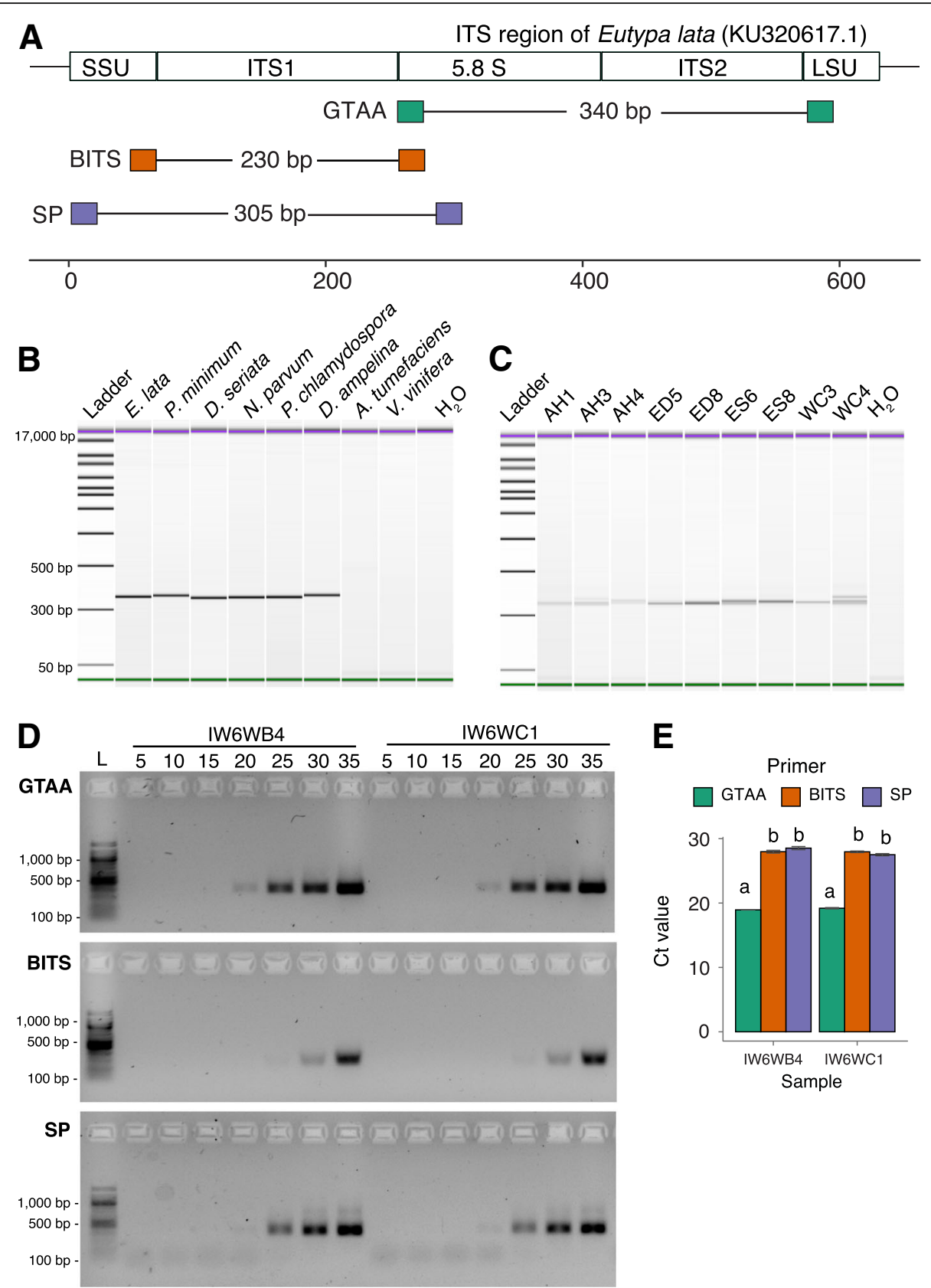

Fig. 1 Primer design and testing. a Schematic representation of the annealing sites of forward and reverse GTAA, BITS, and SP primers in the fungal ribosal ITS. Reported amplicon sizes were calculated based on the ITS sequence of Eutypa lata (KU320617.1) as an example. b Bioanalyzer electropherograms showing PCR amplicons sizes generated using GTAA primers from purified fungal grapevine trunk pathogens: $E$. lata, Phaeoa. minimum, Dip. seriata, N. parvum, Phaeom. chlamydospora, and Dia. ampelina. Agrobacterium tumefaciens, V. vinifera and nuclease-free water were included as controls. c Bioanalyzer electropherograms showing PCR amplicons sizes generated using GTAA primers from selected field samples of mature vines infected with different trunk disease symptoms. AH: apparently healthy, ED: Eutypa Dieback, ES: Esca,WC: wood cankers (no leaf symptoms), and numbering corresponds to different biological replicates (same samples as in Fig. 5). d PCR products of GTAA, BITS, and SP primers from grapevine samples inoculated with N. parvum at six weeks post-inoculation sampled every five-cycles and visualized on an agarose gels. L: 100 bp Ladder. e Cycle thresholds (Ct) measured by qPCR of the same samples shown in (d)

(FN; i.e., not amplified or not assigned), and true positive (TP; i.e., correctly assigned, Fig. 2c) rate. We calculated rates of $\mathrm{FP}, \mathrm{FN}$, and $\mathrm{TP}$ for all combinations of mismatches to estimate the overall performance of each primer set. GTAA primers had the highest sensitivity $\left(\mathrm{TP} /(\mathrm{TP} / \mathrm{FN})^{*} 100=89.50 \pm 6.45 \%\right)$, followed by BITS 


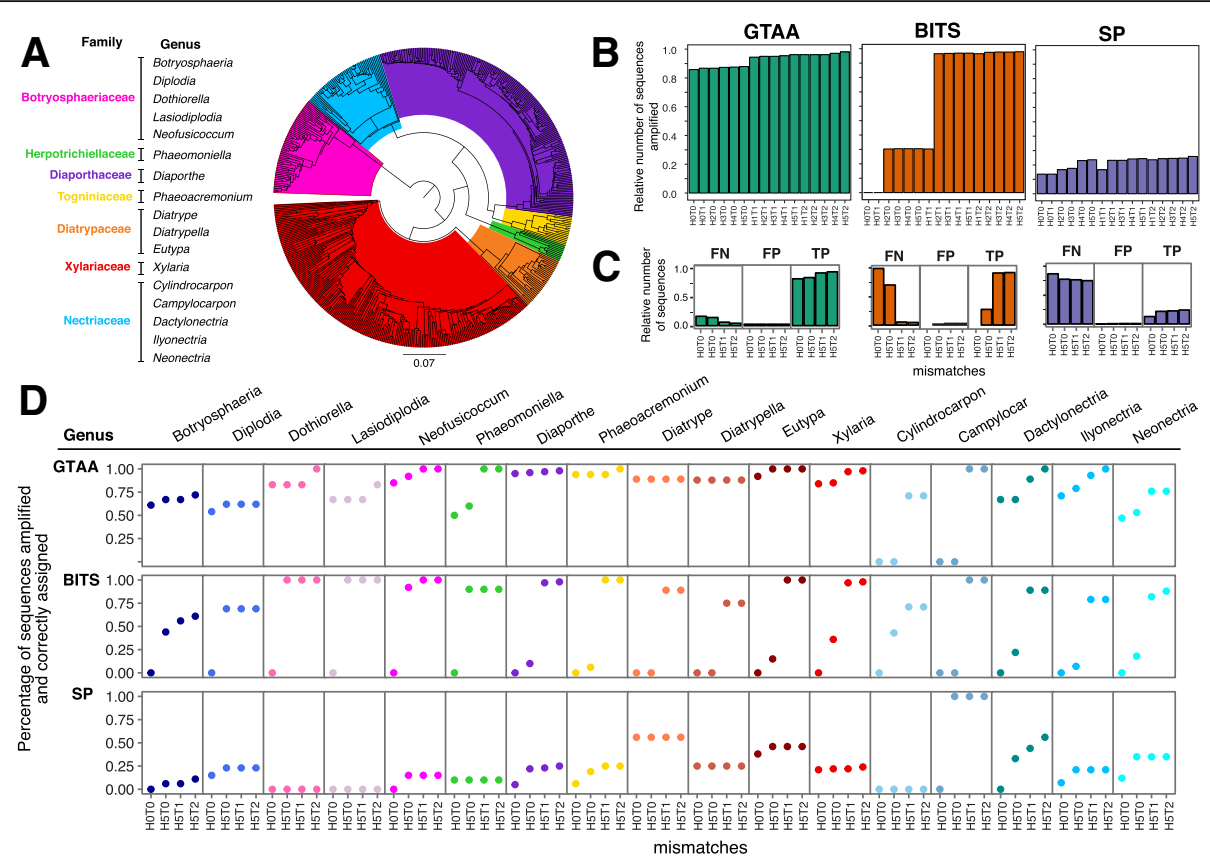

Fig. 2 In silico simulation of amplification and taxonomic assignment. a Neighbor-joining tree of the full-length ITS sequences included in the custom database used in the simulation. $\mathbf{b}$ Barplots showing the number of sequences predicted to be amplified by each primer set at different combinations of mismatches. $\mathrm{H}$ : primer head. T: primer tail. Numbers correspond to the number of mismatches either in $\mathrm{H}$ or T. $\mathbf{c}$ Barplots showing the number of false negative (FN), false positive (FP), and true positive (TP) sequences with each primer set. $\mathbf{d}$ Percentage of sequences per genus correctly assigned with each primer set at the different mismatch combinations

$(54.25 \pm 47.86 \%)$, and SP $(20.50 \pm 2.53 \%)$. SP and GTAA primers displayed similar precision (SP: TP/(TP + FP) $* 100=97.50 \pm 1.00 \%$; GTAA: $97.00 \pm 0.00 \%)$, which was higher than that of BITS primers $(72.25 \pm 48.18 \%)$. The different performance of the three primer sets in the simulation appeared to be mostly due to amplification bias against certain genera (Fig. 2d). GTAA primers amplified and correctly assigned to the proper genera a larger fraction of sequences than the other two primer sets for 14 out of 17 genera tested. This was the case for the following widely distributed trunk pathogens: Eutypa (GTAA: $98.0 \pm 4.0 \%$, BITS: $53.8 \pm 53.8 \%$, and SP: $44.0 \pm$ $4.0 \%$ ), Diaporthe (GTAA: $96.5 \pm 1.3 \%$, BITS: $51.3 \pm$ $53.6 \%$, and SP: $18.7 \pm 9.3 \%$ ), and Phaeoacremonium (GTAA: $95.5 \pm 3.0 \%$, BITS: $51.5 \pm 56.1 \%$, and SP: $18.7 \pm$ 9.0\%). BITS primers correctly assigned more sequences for Lasiodiplodia (GTAA: $71.0 \pm 8.0 \%$, BITS: $75.0 \pm$ $50.0 \%$, and SP: $0.0 \pm 0.0 \%$ ) and Cylindrocarpon (GTAA: $35.5 \pm 41.0 \%$, BITS: $46.3 \pm 33.54 \%$, and SP: $0.0 \pm 0.0 \%$ ). SP primers correctly assigned more sequences for Campylocarpon (GTAA: $50.0 \pm 57.7 \%$, BITS: $50.0 \pm 57.7 \%$, and SP: $75.0 \pm 50.0 \%)$. Overall, this simulation predicted that, unlike the two universal primer sets, GTAA primers amplify ITS of more trunk pathogens and allow taxonomic assignment with greater sensitivity (i.e., higher true positive rate) and specificity (i.e., lower false negative rate). SP primers were not included in further experiments, due to their poor performance in these early stages.

\section{Analysis of mock communities and infection time course} To evaluate the primers for characterizing the species composition of mixed infections, we sequenced mock community samples with an Illumina MiSeq (Fig. 3a-b). Mock samples were created by mixing DNA of apparently healthy grapevine stems and fungal DNA as follows: $90 \%$ grape with $10 \%$ of fungal DNA (E. lata, Phaeoa. minimum, or Phaeom. chlamydospora), $80 \%$ grape with $10 \%$ of each of two fungal isolates, or $70 \%$ grape DNA with $10 \%$ of each of three fungal isolates. Another set of samples were created with equal concentrations of DNA of a total of six species (see more details in Methods and Additional file 3). We tested multiple approaches for taxonomic assignment. Although they all delivered very similar result, the OTU classifier Mothur was selected for further analysis because it overall delivered the greatest correlation between observed and expected values (Additional file 4). Although the DNA was extracted from stems with no symptoms of trunk disease, both primer sets detected 

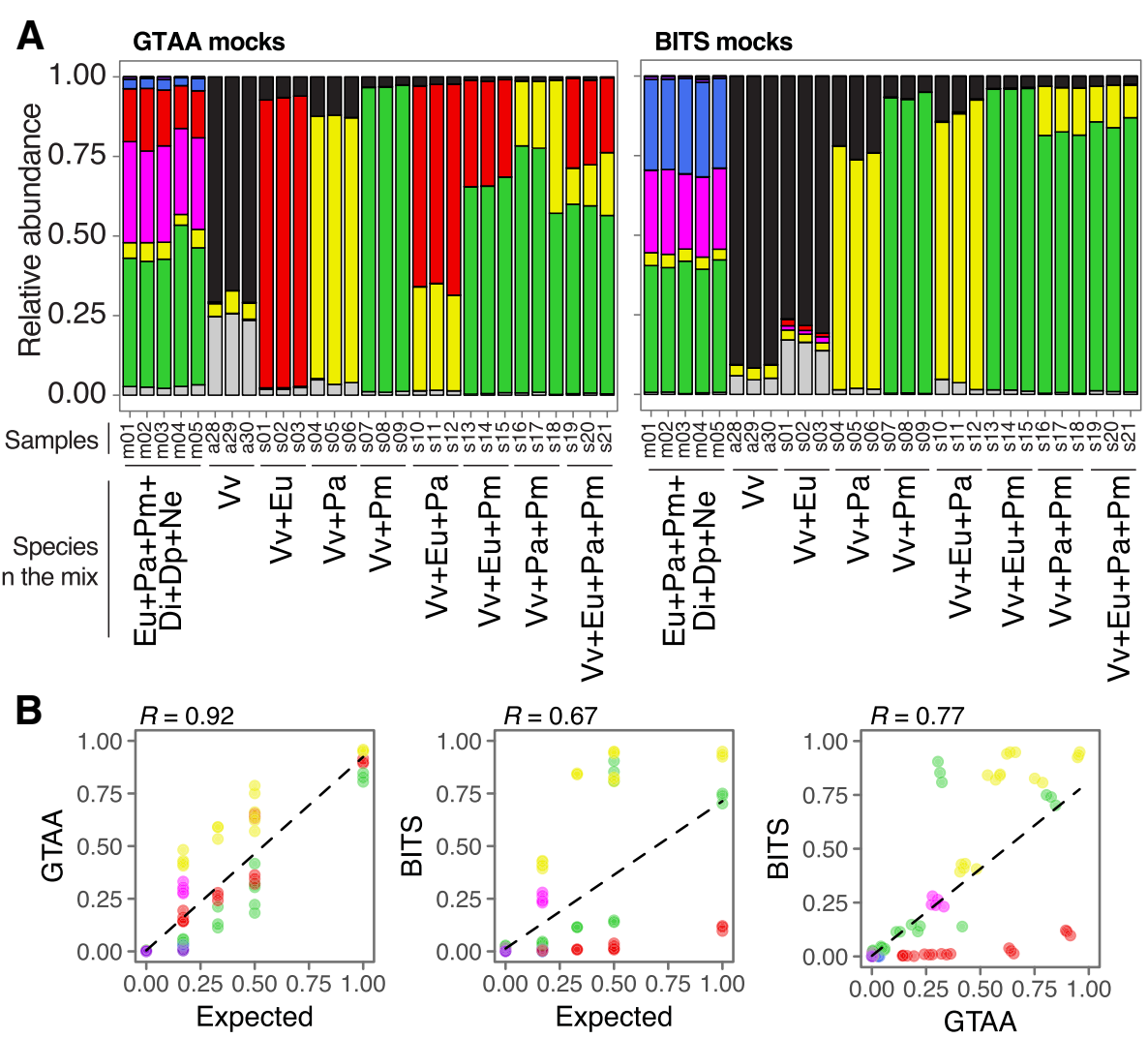

\section{Genera}
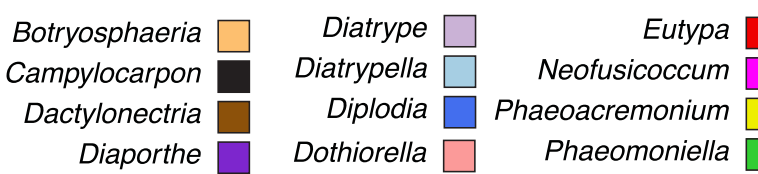

Fig. 3 Results of DNA metabarcoding of mock communities. a Stacked barplots showing the relative abundance of genera in the mock communities with different proportion of fungal species identified using the GTAA and BITS primers. Eu: Eutypa, Pa: Phaeoacremonium, Pm: Phaeomoniella, Di: Diaporthe, Dp: Diplodia, Np: Neofusicoccum, and Vv: Vitis vinifera. b Linear correlations between observed and expected abundances for each genus contained in the mock community. R values correspond to Pearson's correlation coefficients

fungi, mostly belonging to the genera Campylocarpon and Phaeoacremonium (Fig. 3a). In these samples, compared to the GTAA primers, the BITS primers assigned a greater proportion of reads to known taxa, likely because GTAA were designed to target taxa associated with trunk diseases. When grape DNA was mixed with DNAs of Phaeoa. minimum and Phaeom. chlamydospora both primer sets identified the correct taxa, with small relative difference from the expected values [relative difference between expected and observed $(\delta)$ in GTAA $=11.02 \pm$ $7.0 \%$ and in BITS $=16.68 \pm 11.39 \%]$. For mock communities including Eutypa, GTAA primers detected this trunk pathogen in similar amounts to the expected abundance $(\delta=9.74 \pm 1.10 \%)$, whereas BITS primers underestimated its abundance $(\delta=88.87 \pm 1.27 \%)$. In mock communities with equal concentrations of DNA from E. lata, Phaeoa. minimum, Phaeom. chlamydospora, $N$. parvum, D. seriata, and D. ampelina, the correlation of expected and observed abundances in these mock communities was greater for GTAA $(R=0.92)$ than BITS $(R=0.67)$, though there was no significant difference between both primers $(P=0.16$, Fig. 3b; Additional file 5). The BITS primers showed an underrepresentation of Eutypa $(\delta=16.70 \pm 0.12 \%)$, and Phaeoacremonium $(\delta=13.10 \pm 0.63 \%)$, and overrepresentation of Phaeomoniella $(\delta=24.34 \pm 1.56 \%)$ (Fig. 3a). Because DNA was mixed in equal amounts, the expected relative abundance of each genus was $16.6 \%$. GTAA primers detected Eutypa at $16.42 \pm 2.41 \%$, whereas BITS primers detected this trunk pathogen at $0.05 \pm 0.03 \%$. In 
the case of Diplodia, GTAA primers estimated the abundance of the genus at $3.13 \pm 0.48 \%$ and BITS primers at $28.9 \pm 0.8 \%$. Interestingly, neither primer set was able to detect properly Diaporthe, reporting only $0.58 \pm 0.23 \%$ and $0.87 \pm 0.15 \%$ for GTAA and BITS primers, respectively. Nonetheless, GTAA primers provide a better qualitative and quantitative representation of important trunk pathogens.

We then tested the two primers using grape samples collected at different time points after controlled inoculation with a trunk pathogen. The objective of this analysis was to determine if the metabarcoding approach could detect quantitative differences between samples at early and late stages of infection. Neofusicoccum parvum was selected for this analysis as it is a fast growing and aggressive trunk pathogen and, therefore, can be used for studying grapevine stem colonization over a reasonable amount of time. Vines were inoculated with $N$. parvum and stem samples were collected at $24 \mathrm{~h}, 2$ weeks, and 6 weeks post-inoculation. Plants non-inoculated wounded (NIW) and non-inoculated non-wounded (NINW) were included as controls. As expected, Neofusicoccum was predominant in the inoculated wounded (IW) samples, but absent from the controls (Fig. 4), except for a single NIW sample, possibly due to cross-contamination during wounding or from contamination of the propagation material. The two primer sets provided similar fungal composition profiles characterized by five-fold increase in the average percentage of Neofusicoccum between $24 \mathrm{~h}$ and 6 weeks post-inoculation.

Analysis of field samples and comparison with referencebased shotgun metagenome sequencing

We then tested the primers on naturally infected grapevines. We used the same 28 field samples described in [13], which allowed us to compare the metabarcoding approach with the quantitative taxonomic profiles obtained by a reference-based shotgun metagenome sequencing. The samples were grouped according to symptoms into Eutypa dieback (ED), Esca (ES), wood canker without foliar symptoms (WC), and apparently-healthy (AH). All 28 field samples were amplified with both GTAA and BITS primers. A subset of 14 samples was amplified also using SP primers. Taxonomic classification was performed with multiple methods and results compared to the whole-genome metagenomics results, which, at least in case of GTAA, delivered very similar results (see more details in Methods and Additional file 6). Mothur results had the greatest correlation with shotgun metagenome sequencing for both primers and were used for further analyses. In addition to those with genomes in the multispecies reference, taxonomy assignment based on amplicon metabarcoding detected other 14 genera with abundances $>0.05 \%$ in one or more samples. Both GTAA and BITS primer sets identified Alternaria, Cyphellophora, and Penicillium, whereas
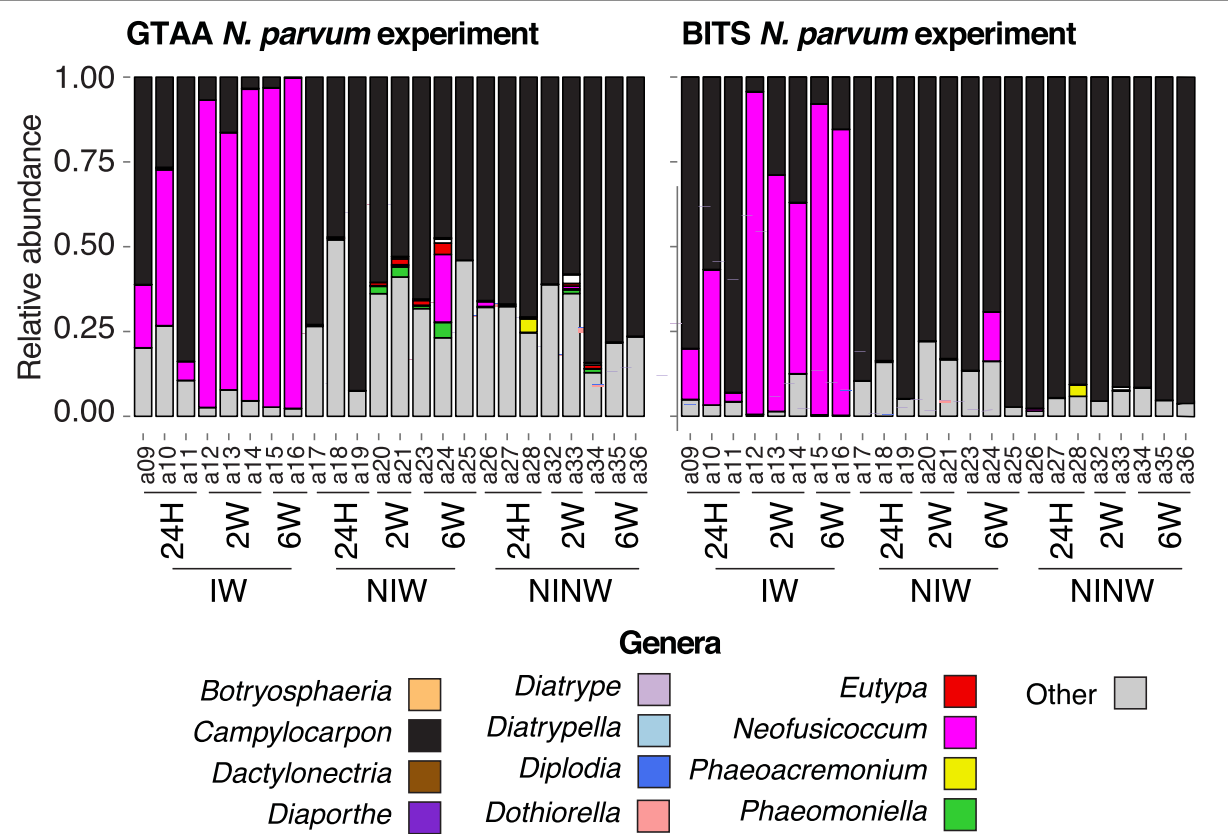

Fig. 4 Results of DNA metabarcoding of an infection time-course. Stacked barplots showing the relative abundance of the genera detected in a time course experiment after inoculation with N. parvum. IW: wound-inoculated with N. parvum; NIW: non-inoculated non-wounded; NINW: noninoculated non-wounded controls 

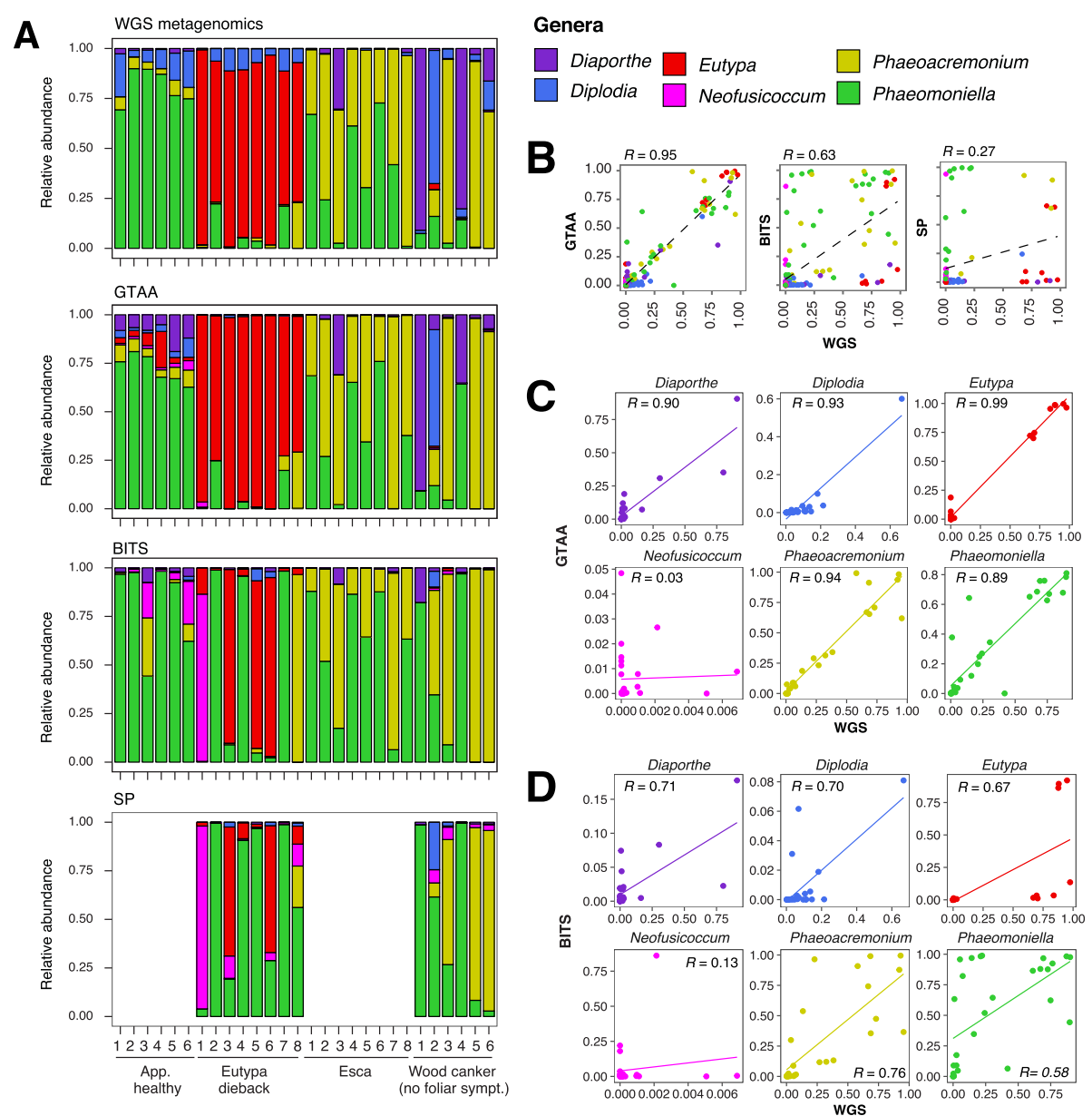

Fig. 5 Results of DNA metabarcoding of field samples and comparisons with shotgun whole-genome metagenomics (WGS). a Stacked barplots showing the relative abundance of the genera detected by WGS and DNA metabarcoding with GTAA, BITS, and SP primers. The 28 samples were collected from mature vines in the field with the following trunk disease symptoms: apparently healthy (no foliar or wood symptoms), Eutypa Dieback, Esca and wood cankers (no leaf symptoms). The numbering corresponds to different biological replicates grouped by the disease symptoms displayed by the grapevines from where they were collected. b Scatter plots showing the correlations of the relative abundance obtained by DNA metabarcoding and WGS. c Scatter plots showing the correlations of the relative abundance separately for each genus obtained by DNA metabarcoding using the GTAA primers and WGS. d Scatter plots showing the correlations of the relative abundance separately for each genus obtained by DNA metabarcoding using the BITS primers and WGS. R values correspond to Pearson's correlation coefficients

Cladosporium, Aureobasidium, Gibberella, and Cryptovalsa were only identified by GTAA primers, and Angustimassarina, Exophiala, Erysiphe, Meyerozyma, Acremonium, and Vishniacozyma by BITS primers. GTAA primers revealed species abundances at very similar levels to those obtained by metagenomics analysis (Fig. 5a), with a strong linear correlation between the two approaches $(R=0.95$; Fig. $5 \mathrm{~b})$, which was higher than those of both BITS $(R=0.63)$ and SP primers $(R=0.27)$. In agreement with the other results described above, BITS primers underestimated Eutypa in Eutypa-dieback samples and overrepresented Phaeomoniella in Esca samples. The even weaker correlation obtained with SP primers was due to the strong bias against Eutypa and Diaporthe. Surprisingly, Diaporthe, which was detected at much lower level than expected in the mock communities, showed a good correlation between metabarcoding and metagenomic results with both GTAA $(R=0.90)$ and BITS $(R=0.71)$ primers (Fig. $5 \mathrm{c} \&$ d). This inconsistency confirms the difficulty in resolving this genus [59]. GTAA primers showed stronger correlations across all genera of trunk pathogens represented in the multi-genome reference $(0.89<R<0.99$, Fig. 5 c $)$ compared to those of BITS $(0.58<R<0.75)$. Both primer sets overestimated Neofusicoccum, potentially because of the presence of other Botryosphaeriaceae, whose sequences may have been wrongly assigned to Neofusicoccum as ITS has been shown to poorly differentiate the species in this 
family [59]. Overall, our findings confirm the universal primers have a significant bias against important taxa and were outperformed by our GTAA primers for trunk pathogens.

\section{Discussion}

In this study, we tested the application of DNA metabarcoding to profile the fungal taxa associated with grapevine trunk diseases. We show that DNA metabarcoding of ribosomal ITS amplified with commonly-adopted universal primers consistently misrepresented the abundance of important trunk pathogen species, such as Eutypa and Phaeomoniella. The customization of primer design using trunk pathogen sequences as template led to improve the results with greater sensitivity. This was likely due to greater sequence similarity between the GTAA primers and the ITS of the grapevine trunk pathogens they target. On average the sequence identity of the grapevine trunk pathogen targets was significantly greater with the GTAA primers $(97.4 \pm 5.5 \% ; P<2 \mathrm{e}-16)$ than with the other universal primers (BITS: $90.2 \pm 7.1 \%$; SP: $83.3 \pm 0.2 .2 \%)$ used in the study. The lower identity of the universal primers was likely responsible for the bias of the universal primers against important trunk pathogen taxa. Amplification bias of universal ITS primers due to higher levels of mismatches for certain taxonomic group has been observed in previous studies [35, 60-63]. Importantly, we also showed that the GTAA primers had higher sensitivity while maintaining a precision threshold for taxonomic assignment of 97\%, suggesting that the choice of the target region within the ITS, in this case the ITS2, also played a role in improving the DNA metabarcoding for these organisms. We should stress out that BITS and SP primers are not the only available universal primers and the goal of this study was not to provide a comprehensive survey of all universal ITS metabarcoding primers. BITS and SP were selected, because they are both widely used DNA barcoding primers, including in studies conducted on vineyard and wine must samples [49-54]. We cannot rule out that other universal primers targeting the ITS1 or ITS2 regions that were not tested in this study may have performed differently. However, the results presented in this study show that universal primers may not be always appropriate to study a fungal community and, when fungal community composition is available, researchers should consider customizing their DNA metabarcoding primers. In addition, we illustrate the value of assessing both the amplification and taxonomy usefulness of the metabarcoding primers in silico prior to downstream wet lab evaluations.

In addition to customization of primers, the inclusion of other gene targets as additional DNA barcodes should help overcome some of the limitations associated with the ITS region, such as copy number variation between and within species and low resolution in separating some phylogenetically closely related fungal species [45, 64]. For example, the ITS region does not accurately identify species of plant-pathogenic fungi like Alternaria, Botryosphaeria, and Diaporthe [59]. The genera for which the GTAA primers consistently underestimated abundances like Lasiodiplodia, Botryosphaeria, Diplodia, and Diaporthe are known to be difficult to be resolved with the ITS region alone [59]. The high correlation between metagenomics and metabarcoding results using the GTAA primers suggest that copy number variation of the ITS region is not an overwhelming issue for the grapevine trunk pathogens present in the field samples.

Nonetheless, we expect that the inclusion of additional barcodes, such as $\beta$-tubulin and elongation factor $1-\alpha$, will help increase accuracy of taxonomic identification at the species level and help measure those genera for which the ITS is known not to be effective $[20,23,65,66]$.

\section{Conclusions}

As trunk diseases are complex diseases caused by mixed infections, DNA metabarcoding should provide a rapid and effective method for high-throughput multispecies profiling, overcoming the limitations of currently applied methods. Universal primers are advantageous in exploratory analysis where a priori knowledge on the taxonomic composition of the samples is limited or not available. However, a more targeted approach should be used when the objective is to profile a more defined group of microorganisms, like the grapevine trunk pathogens which symptoms have been consistently associated with certain fungal species $[4,20,67]$. Overall, the results presented here demonstrated that DNA metabarcoding can be applied to grapevine trunk diseases. With further improvement of taxonomic identification by combining multiple barcoding loci and of quantification by measurement of direct correlation between fungal biomass and PCR amplification cycles, we envision DNA metabarcoding to be routinely applied in trunk pathogen research and profiling. DNA metabarcoding provides multiple advantages to methods employed in the past. Namely, there is no need of fungal isolation, it allows high number of samples to be analyzed at the same time given the multiplexing potential of the technology, and takes advantage of the constantly improving high-throughput sequencing technologies. Since wood pathogens may remain asymptomatic in young, non-stressed vines, propagation material may contain latent fungal infections and may become symptomatic after planting and serve as a source of inoculum for further infections of potentially clean plants. By allowing the rapid testing of large number of wood samples 
from mother plants in foundation blocks and propagation material in nurseries, we expect that the applications of metabarcoding to trunk pathogen profiling will help reduce the amount of trunk pathogens introduced into vineyards at planting as well as the incidence of young vine decline. Our results also demonstrated that primer customization and testing are crucial to ensure the validity of DNA metabarcoding results.

\section{Methods}

Metabarcoding primers targeting grapevine trunkassociated ascomycetes (GTAA)

Ribosomal Internal transcribed spacer (ITS) sequences of trunk pathogens and other wood-colonizing fungi of grape, specifically in the Division Ascomycota, were retrieved from the TrunkDiseaseID.org database [20]. Sequences were aligned using ClustalW2 (v2.1; [68]) to identify conserved regions. Sequence alignments of the conserved regions were used as input for the metabarcoding primer design software Primer Prospector v1.0.1 [69], using a sensitivity threshold of $80 \%$ and an initial primer seed size of $5 \mathrm{bp}$. The ITS sequence of $E$. lata (GeneBank KU721859.1) was used as a 'reference'. The final primer set was selected based on median amplicon size, and mismatches, gaps, and numbers of matches to the sequences in the database. The base pairs 'AG' were used as a linker between the primer and an eight-nucleotide barcodes on the $5^{\prime}$ region of the forward primer sequence. Barcode sequences were as described in [70]. A list of barcoded forward GTAA primers is listed in Additional file 7.

A custom database was compiled with full length ITS sequences of species in the following genera commonly isolated from grapevines: Botryosphaeria, Diplodia, Dothiorella, Lasiodiplodia, Neofusicoccum, Phaeomoniella, Diaporthe, Phaeoacremonium, Diatrype, Diatrypella, Eutypa, Xylaria, Cylindrocarpon, Campylocarpon, Dactylonectria, Ilyonectria, and Neonectria. Sequences were retrieved from the NCBI GenBank repository. Completeness of the ITS sequences was validated using the hidden Markov models-based software ITSx [71]. Only sequences spanning the entire ITS region (ITS1, $5.8 \mathrm{~S}$, and ITS2) were kept for downstream analysis. Species and GenBank accessions of the complete ITS sequences included in the custom database are listed in Additional file 2. To reduce redundancy and identify outliers, the complete ITS sequences were clustered using the UCLUST algorithm [72] integrated in QIIME (v1.9.1; [73]) with 97\% identity. The longest representative sequence of each cluster was selected, using the QIIME 'pick_rep_set.py' function. All representative sequences were aligned using Mafft v7.271 [74]) with the '--auto' argument and 1000 iterations. Sequences clustering outside the expected family were removed from the final custom database.

The program Degenerate In-Silico PCR (dispr, https:// github.com/douglasgscofield/dispr) was used to predict and evaluate the amplification of sequences of the custom ITS database, using our GTAA primers, and universal BITS [48], and SP [49] primers. Dispr allowed an amplicon size of 100 to $400 \mathrm{bp}$, all combinations up to five mismatches in the head of the primer (' $\mathrm{H}$ ' or 5 '-most region), and all combinations up to two mismatches in the tail of the primer (' $\mathrm{T}$ ' or the remaining 3 '-end of the primer). The resulting amplicons produced in silico were then used for taxonomy assignment with $80 \%$ confidence, using Mothur (v1.39.5; [58]), as it is integrated in QIIME (v1.9.1). The UNITE database v7.2 [75] was used as taxonomic reference. True positives were defined as sequences that were assigned to the expected genus, false positives were sequences assigned to a different genus, and false negatives were sequences not assigned to any genus or were not amplified by dispr.

To test primer affinity, quantitative real time PCR (qPCR) was performed using a QuantStudio 3 instrument (Applied Biosystems). Each $15 \mu \mathrm{l}$ reaction mixture contained $2 \mathrm{ng}$ of gDNA, $0.3 \mu \mathrm{M}$ each primer and, $1 \mathrm{X}$ Power SYBR green PCR Master Mix (Applied Biosystems). The PCR stage included a first denaturation step $\left(95^{\circ} \mathrm{C} 10 \mathrm{~min}\right)$, followed by 40 cycles of $95^{\circ} \mathrm{C}(15 \mathrm{~s})$ and $60^{\circ} \mathrm{C}(1 \mathrm{~min})$.

To generate mock communities, we combined (i) DNA from a healthy grapevine with DNA from pure cultures of three trunk pathogens at different concentrations, or (ii) equal concentrations of DNA from pure cultures of six trunk pathogens. For the former, grape and fungal DNA were combined as follows: $90 \%$ grape with $10 \%$ E. lata isolate Napa209 [76], Phaeoa. minimum isolate 1119 [77], or Phaeom. chlamydospora isolate C42 [78]; 80\% grape with 10\% of each of two fungal isolates (in all three pair-wise combinations of the three isolates); and $70 \%$ grape DNA with $10 \%$ of each of the three fungal isolates. For the latter, equal concentrations of DNA were combined from the same three trunk pathogens and three additional species: $N$. parvum isolate UCD646So [11], Dia. ampelina isolate Wolf911 [79], and Diplodia seriata isolate SBen831 [80]. Grape DNA was extracted from the stems of a non-inoculated, non-wounded plant; this DNA template came from a previous experiment [81]. The mock communities containing grape DNA were amplified and sequenced as three independent samples, whereas the mock community of six fungal DNAs was amplified and sequenced as five independent samples. The fungi used to prepare the mock communities were grown on Potato Dextrose Agar (PDA; Difco laboratories, Detroit, MI). DNA was 
extracted as described in [73] and measured with a fluorometer (Qubit, Life technologies). To test in planta detection of a trunk pathogen at variable levels of infection (i.e., from low to high concentrations of fungal biomass over time), DNAs for the infection time course of $N$. parvum were extracted from the same samples described in [81]. Briefly, 1-year-old potted $V$. vinifera 'Cabernet Sauvignon' FPS 19 plants were inoculated with isolate UCD646So mycelia. Woody stems were collected at seven time points: $0 \mathrm{~h}$ post inoculation (hpi), $3 \mathrm{hpi}, 24$ hpi, 2 weeks post inoculation (wpi), 6 wpi, 8 wpi, and 12 wpi. Wood samples from $1 \mathrm{~cm}$ below the inoculation site were collected using flame-sterilized forceps and immediately placed in liquid nitrogen for nucleic acids extraction. Infections were confirmed by positive recovery of the pathogen after 5-day growth on PDA.

To test in planta detection of multiple trunk pathogens in mixed infection (i.e., to characterize the species composition of a naturally established trunk-pathogen community), DNA from the same 28 field samples described in [13] was used to make cross-technology comparisons. These field samples were collected from mature vines ( $>8$ years-old) showing a variety of the most common symptoms associated with trunk diseases. Wood samples were collected from distinct plants with the following combinations of symptoms: Eutypa dieback foliar and wood symptoms, Esca foliar and wood symptoms, wood symptoms and no foliar symptoms, and apparently healthy plants with no foliar or wood symptoms.

\section{High throughput sequencing libraries}

Each sample was amplified using the unique 8-nt barcode forward primer sets for GTAA and BITS, to enable sample multiplexing. The $25-\mu \mathrm{l}$ PCR reaction mix contained 2 ng of DNA template, 1X Colorless GoTaq flexi buffer (Promega Corporation, Madison, WI), $1.5 \mathrm{mM}$ $\mathrm{MgCl}_{2}, 0.1 \mathrm{mg} / \mathrm{ml} \mathrm{BSA}, 0.2 \mathrm{mM}$ dNTPs, $0.4 \mu \mathrm{M}$ of each primer, and 1.25 units of GoTaq Flexi DNA polymerase (Promega Corporation, Madison, WI). PCR program (Veriti thermal cycler, Applied Biosystems) was as follows: initial denaturation at $94^{\circ} \mathrm{C}$ for $3 \mathrm{~min}$, followed by 35 cycles at $94^{\circ} \mathrm{C}$ for $45 \mathrm{~s}, 55^{\circ} \mathrm{C}$ for $1 \mathrm{~min}$., and $72{ }^{\circ} \mathrm{C}$ for $1 \mathrm{~min}$., and a final extension at $72^{\circ} \mathrm{C}$ for $10 \mathrm{~min}$. In the experiments to assess primer affinity, reactions were stopped after $5,10,15,20,25,30$, and 35 cycles. Following PCR, amplicon size and uniqueness were verified using gel electrophoresis, and bands were cleaned using Ampure XP magnetic beads (Agencourt, Beckman Coulter). DNA concentration was determined for each purified amplicon using Qubit (Life technologies). For the single isolate validation, amplicons were sequenced with Sanger (DNA Sequencing Facility, University of California, Davis).
For high-throughput sequencing, equimolar amounts of all barcoded amplicons were pooled into a single sample, the total concentration of which was determined by Qubit. Five hundred nanograms of pooled DNA were then end-repaired, A-tailed and single-index adapter ligated (Kapa LTP library prep kit, Kapa Biosystems). After adapter ligation, the sample was size-selected with two consecutive $1 \mathrm{X}$ bead-based cleanups; concentration and size distribution were determined with Qubit and Bioanalyzer (Agilent Technologies), respectively. DNA libraries were submitted for sequencing in 250-bp paired-end mode on an Illumina MiSeq (UCDavis Genome Center DNA technologies Core). All FASTQ files with the amplicon sequences separated by barcode were deposited in the NCBI Sequence Read Archive (BioProject: PRJNA485180; SRA accession: SRP156804).

\section{Amplicon sequencing community analysis}

Adapter-trimming was carried out using BBDuk (BBMap v.35.82; http://jgi.doe.gov/data-and-tools/bb-tools/) in paired-end mode with sequence "AGATCGGAAG" and the following parameters: $k$ trim $=\mathrm{r}, \mathrm{k}=10, \operatorname{mink}=6$, edist $=2, \quad$ ordered $=\mathrm{t}, \quad$ qtrim $=\mathrm{f}$ and minlen $=150$. Adapter-trimmed FASTQ files were then quality-filtered using Trimmomatic v0.36 [82] with paired-end mode, phred33, a sliding windows of 4:19, and a minimum length of $150 \mathrm{bp}$. Sequencing data were then processed in the QIIME environment v1.9.1 [73]. Barcodes were extracted from the FASTQ files using the "extract_barcodes.py" function with the "-a" argument that attempts read orientation and a barcode length of eight base pairs. The resulting sequences and barcodes were used to tag the reads with "split_libraries_fastq.py", a threshold quality score of 20, and a barcode size of eight basepairs. Operational taxonomic units (OTUs) were identified with a 99\% similarity threshold using the UCLUST algorithm [72] with the reverse strand match enabled ("-z"), and the longest sequence of each OTU was chosen as representative sequence. Taxonomy assignment was carried out using Mothur (v1.39.5; [58] with the UNITE database v7.2 [75] or the custom database as reference with a $80 \%$ confidence threshold). Two additional OTU classifiers, BLAST and UCLUST, as implemented in QIIME were also used to analyze mock samples and field samples for taxonomy assignment testing inside QIIME (Additional files 4 and 6). For each sample, sequences were randomly sampled with the function "single_rarefaction.py" from the OTU tables to obtained a total number of sequences per sample equal to the lowest number of reads across GTAA, BITS, and SP datasets. In the mock and $N$. parvum artificially inoculated samples, the lowest value corresponded to 1119 reads of the sample "a33" from the GTAA primers. In the field samples the value corresponded to 23,609 reads of sample 
"ED6" from the BITS primers. The Taxonomy tables at the genus level were then created using "summarize_taxa.py". Taxonomic assignments based on OTUs generated in QIIME were compared with results obtained by BLASTn (v2.2.31+) alignments of each individual read to the custom ITS database. We considered as hits only those alignments that covered more than $97 \%$ of the query sequence with sequence identity greater than $97 \%$. The sequence with the highest identity was chosen as the best hit for each query sequence. The results were compared to the expected values and presented in Additional file 4 .

\section{Additional files}

Additional file 1: Text S1. Assembled amplicon sequences produced by the GTAA primers. Amplicons were sequenced with Sanger. Each sequence description includes the GenBank accession number to the best hit of the custom database created in this study as well as identity and coverage of the alignment. (FASTA $2 \mathrm{~kb}$ )

Additional file 2: Table S1. Table with GenBank accession numbers and sequences retrieved from the NCBI used as a database for primer evaluation. TP: True Positive, FP: False Positive and FN: False Negative. (XLSX $124 \mathrm{~kb})$

Additional file 3: Table S2. Table with the proportions of fungal DNA of pure cultures of six grapevine trunk pathogens mixed to create the mock samples. Samples m01 to m05 were created with a mix of equal proportions of the six species. Samples s01 to s21 were created with a mix of $10 \%$ of one or more species, and a remaining percentage of grape DNA. The values presented are the expected proportions of fungal DNA. (XLSX $9 \mathrm{~kb})$

Additional file 4: Figure S1. Scatterplots showing the correlation between expected relative abundance based on how mock communities were prepared and the observed relative abundance of fungal taxa detected in the mock communities using the GTAA and BITS primers. The genus abundances resulting from the QIIME pipeline with Mothur, BLASTn and UCLUST taxonomy classifiers, as well as direct BLASTn of the reads to the custom database created in this study, were compared to the expected values. $R$ values correspond to Pearson's correlation coefficients. (PDF $643 \mathrm{~kb}$ )

Additional file 5: Figure S2. Scatterplots showing the correlation between expected relative abundances and observed values using GTAA and BITS primers for individual fungal taxa in the mock communities. $R$ values correspond to Pearson's correlation coefficients. (PDF $286 \mathrm{~kb}$ )

Additional file 6: Figure S3. Scatterplots showing the correlation of relative abundance of fungal taxa detected using GTAA and BITS, and whole-genome shotgun metagenomics results with multiple taxonomy classifiers. The genus abundances resulting from the QIIME pipeline with Mothur, BLASTn, and UCLUST, were compared to the metagenomics values. Mothur results were also compared to the other classifying methods. R values correspond to Pearson's correlation coefficients. (PDF $634 \mathrm{~kb})$

Additional file 7: Table S3. List of forward GTAA primers with linker and barcode sequences. (XLSX $46 \mathrm{~kb}$ )

\section{Acknowledgements}

Not applicable

\section{Funding}

This work was supported by the American Vineyard Foundation (grants 2016-1798, 2017-1798, 2018-1798) and the USDA, National Institute of Food and Agriculture, Specialty Crop Research Initiative (grant 2012-51181- 19954). DC was also supported by the Louis P. Martini Endowment in Viticulture. AMC was partially supported by the Horace O. Lanza Scholarship, Wine Spectator Scholarship, The Pearl \& Albert J. Winkler Scholarship in Viticulture, and the Summer Graduate Student Researcher Award at UC Davis to support graduate research in engineering, computer sciences, and disciplines with engineering-related applications and methods.

\section{Availability of data and materials}

All FASTQ files with the amplicon sequences separated by barcode were deposited in the NCBI Sequence Read Archive (BioProject: PRJNA485180).

\section{Authors' contributions}

Conceived and designed the experiments: AMC and DC. Performed the lab experiments: RFB, AMC, ET, and JD. Performed field sampling: PER. Performed bioinformatic analysis: AMC. Wrote the manuscript: AMC, KB, and DC. All authors read and approved the final manuscript.

Ethics approval and consent to participate

Not applicable

\section{Consent for publication}

Not applicable

\section{Competing interests}

The authors declare that they have no competing interests.

\section{Publisher's Note}

Springer Nature remains neutral with regard to jurisdictional claims in published maps and institutional affiliations.

\section{Author details}

'Department of Viticulture and Enology, University of California Davis, One Shields Ave, Davis, CA 95616, USA. ${ }^{2}$ Department of Botany and Plant Sciences, University of California, Riverside, CA 92521, USA. ${ }^{3}$ United States Department of Agriculture, Agricultural Research Service, Crops Pathology and Genetics Research Unit, Davis, CA 95616, USA.

Received: 16 August 2018 Accepted: 15 November 2018

Published online: 14 December 2018

\section{References}

1. Munkvold G, Duthie J, Marois J. Reductions in yield and vegetative growth of grapevines due to Eutypa dieback. Phytopathology. 1994:84(2):186-92.

2. Siebert JB. Eutypa: The economic toll on vineyards. Wines and Vines. 2001; (April):50-6.

3. Kaplan J, Travadon R, Cooper M, Hillis V, Lubell M, Baumgartner K Identifying economic hurdles to early adoption of preventative practices: the case of trunk diseases in California winegrape vineyards. Wine Econ Policy. 2016;5(2):127-41.

4. Bertsch C, Ramírez-Suero M, Magnin-Robert M, Larignon P, Chong J, AbouMansour E, Spagnolo A, Clément C, Fontaine F. Grapevine trunk diseases: complex and still poorly understood. Plant Pathol. 2013;62(2):243-65.

5. Whitelaw-Weckert M, Rahman L, Appleby L, Hall A, Clark A, Waite H, Hardie W. Co-infection by Botryosphaeriaceae and Ilyonectria spp. fungi during propagation causes decline of young grafted grapevines. Plant Pathol. 2013: 62(6):1226-37.

6. Graniti A, Bruno G, Sparapano L. Three-year observation of grapevines cross-inoculated with esca-associated fungi. Phytopathol Mediterr. 2001; 40(3):376-86.

7. Pierron RJ, Pouzoulet J, Couderc C, Judic E, Compant S, Jacques A. Variations in early response of grapevine wood depending on wound and inoculation combinations with Phaeoacremonium aleophilum and Phaeomoniella chlamydospora. Front Plant Sci. 2016;7:268.

8. Cabral A, Rego C, Nascimento T, Oliveira H, Groenewald JZ, Crous PW. Multigene analysis and morphology reveal novel llyonectria species associated with black foot disease of grapevines. Fungal Biol. 2012;116(1):62-80.

9. Halleen F, Fourie PH, Crous PW. A review of black foot disease of grapevine. Phytopathol Mediterr. 2006:45(4):55-67.

10. Moller W, Kasimatis A, Kissler J. A dying arm disease of grape in California [Eutypa armeniacae]. Plant Dis Reporter. 1974;58(10):869-71.

11. Úrbez-Torres J, Leavitt G, Voegel T, Gubler W. Identification and distribution of Botryosphaeria spp. associated with grapevine cankers in California. Plant Dis. 2006;90(12):1490-503. 
12. Lamichhane JR, Venturi $V$. Synergisms between microbial pathogens in plant disease complexes: a growing trend. Front Plant Sci. 2015;6:385.

13. Morales-Cruz A, Allenbeck G, Figueroa-Balderas R, Ashworth VE, Lawrence DP, Travadon R, Smith RJ, Baumgartner K, Rolshausen PE, Cantu D. Closedreference metatranscriptomics enables in planta profiling of putative virulence activities in the grapevine trunk disease complex. Mol Plant Pathol. 2018;19(2):490-503.

14. Gramaje D, Armengol J. Fungal trunk pathogens in the grapevine propagation process: potential inoculum sources, detection, identification, and management strategies. Plant Dis. 2011;95(9):1040-55.

15. Fourie $P$, Halleen F. Occurrence of grapevine trunk disease pathogens in rootstock mother plants in South Africa. Australas Plant Pathol. 2004;33(2):313-5.

16. Halleen F, Crous R, Petrin O. Fungi associated with healthy grapevine cuttings in nurseries, with special reference to pathogens involved in the decline of young vines. Australas Plant Pathol. 2003;32(1):47-52.

17. Sosnowski MR, Wicks TJ, Scott ES. Control of Eutypa dieback in grapevines using remedial surgery. Phytopathol Mediterr. 2011;50(4):277-84.

18. Rolshausen PE, Úrbez-Torres JR, Rooney-Latham S, Eskalen A, Smith RJ, Gubler WD. Evaluation of pruning wound susceptibility and protection against fungi associated with grapevine trunk diseases. Am J Enol Vitic. 2010;61(1):113-9.

19. Gramaje D, Úrbez-Torres JR, Sosnowski MR. Managing grapevine trunk diseases with respect to etiology and epidemiology: current strategies and future prospects. Plant Dis. 2018;102(1):12-39.

20. Lawrence DP, Travadon R, Nita M, Baumgartner K. TrunkDiseaselD. Org: a molecular database for fast and accurate identification of fungi commonly isolated from grapevine wood. Crop Prot. 2017;102:110-7.

21. Pouzoulet J, Mailhac N, Couderc C, Besson X, Daydé J, Lummerzheim M, Jacques A. A method to detect and quantify Phaeomoniella chlamydospora and Phaeoacremonium aleophilum DNA in grapevine-wood samples. Appl Microbiol Biotechnol. 2013;97(23):10163-75.

22. Pouzoulet J, Rolshausen PE, Schiavon M, Bol S, Travadon R, Lawrence DP, Baumgartner K, Ashworth VE, Comont G, Corio-Costet M-F. A method to detect and quantify Eutypa lata and Diplodia seriata-complex DNA in grapevine pruning wounds. Plant Dis. 2017;101(8):1470-80.

23. Úrbez-Torres J, Haag P, Bowen P, Lowery T, O'Gorman D. Development of a DNA macroarray for the detection and identification of fungal pathogens causing decline of young grapevines. Phytopathology. 2015;105(10):1373-88.

24. Martín MT, Cobos R, Martín L, López-Enríquez L. Real-time PCR detection of Phaeomoniella chlamydospora and Phaeoacremonium aleophilum. Appl Environ Microbiol. 2012;78(11):3985-91.

25. Lawrence DP, Travadon R, Baumgartner K. Novel Seimatosporium species from grapevine in northern California and their interactions with fungal pathogens involved in the trunk-disease complex. Plant Dis. 2018;102(6):1081-92.

26. Pancher M, Ceol M, Corneo PE, Longa CMO, Yousaf S, Pertot I, Campisano A. Fungal endophytic communities in grapevines (Vitis vinifera L.) respond to crop management. Appl Environ Microbiol. 2012;78(12):4308-17.

27. Bruez E, Vallance J, Gerbore J, Lecomte P, Da Costa J-P, Guerin-Dubrana L, Rey P. Analyses of the temporal dynamics of fungal communities colonizing the healthy wood tissues of esca leaf-symptomatic and asymptomatic vines. PLoS One. 2014;9(5):e95928.

28. Bruez E, Baumgartner K, Bastien S, Travadon R, Guérin-Dubrana L, Rey P. Various fungal communities colonise the functional wood tissues of old grapevines externally free from grapevine trunk disease symptoms. Aust J Grape Wine Res. 2016;22(2):288-95.

29. Billones-Baaijens R, Úrbez-Torres JR, Liu M, Ayres M, Sosnowski M, Savocchia S. Molecular methods to detect and quantify Botryosphaeriaceae Inocula associated with grapevine dieback in Australia. Plant Dis. 2018;102(8):1489-99.

30. Agustí-Brisach C, Mostert L, Armengol J. Detection and quantification of Ilyonectria spp. associated with black-foot disease of grapevine in nursery soils using multiplex nested PCR and quantitative PCR. Plant Pathol. 2014; 63(2):316-22

31. Schmidt P-A, Bálint M, Greshake B, Bandow C, Römbke J, Schmitt I. Illumina metabarcoding of a soil fungal community. Soil Biol Biochem. 2013;65:128-32.

32. Whipps J, Hand P, Pink D, Bending GD. Phyllosphere microbiology with special reference to diversity and plant genotype. J Appl Microbiol. 2008; 105(6): 1744-55.

33. Fierer N, Leff JW, Adams BJ, Nielsen UN, Bates ST, Lauber CL, Owens S, Gilbert JA, Wall DH, Caporaso JG. Cross-biome metagenomic analyses of soil microbial communities and their functional attributes. Proc Natl Acad Sci. 2012;109(52):21390-5.

34. Prober SM, Leff JW, Bates ST, Borer ET, Firn J, Harpole WS, Lind EM, Seabloom EW, Adler PB, Bakker JD. Plant diversity predicts beta but not alpha diversity of soil microbes across grasslands worldwide. Ecol Lett. 2015; 18(1):85-95.

35. Lindahl BD, Nilsson RH, Tedersoo L, Abarenkov K, Carlsen T, Kjøller R, Kõljalg U, Pennanen T, Rosendahl S, Stenlid J. Fungal community analysis by highthroughput sequencing of amplified markers-a user's guide. New Phytol. 2013;199(1):288-99.

36. Xu X, Passey T, Wei F, Saville R, Harrison RJ. Amplicon-based metagenomics identified candidate organisms in soils that caused yield decline in strawberry. Horticulture Res. 2015;2:15022.

37. Abdelfattah A, Cacciola SO, Mosca S, Zappia R, Schena L. Analysis of the fungal diversity in citrus leaves with greasy spot disease symptoms. Microb Ecol. 2017;73(3):739-49.

38. Deyett E, Roper MC, Ruegger P, Yang J-I, Borneman J, Rolshausen PE. Microbial landscape of the grapevine Endosphere in the context of Pierce's disease. Phytobiomes. 2017;1(3):138-49.

39. Malacrinò A, Schena L, Campolo O, Laudani F, Mosca S, Giunti G, Strano CP, Palmeri V. A metabarcoding survey on the fungal microbiota associated to the olive fruit fly. Microb Ecol. 2017;73(3):677-84.

40. Quaedvlieg W, Groenewald J, de Jesús Y-MM, Crous P. DNA barcoding of Mycosphaerella species of quarantine importance to Europe. Persoonia. 2012;29:101.

41. Bulman S, McDougal R, Hill K, Lear G. Opportunities and limitations for DNA metabarcoding in Australasian plant-pathogen biosecurity. Australas Plant Pathol. 2018;47(5):467-74.

42. Hodgetts J, Ostojá-Starzewski JC, Prior T, Lawson R, Hall J, Boonham N. DNA barcoding for biosecurity: case studies from the UK plant protection program. Genome. 2016;59(11):1033-48.

43. Taberlet $\mathrm{P}$, Coissac E, Pompanon F, Brochmann C, Willerslev E. Towards next-generation biodiversity assessment using DNA metabarcoding. Mol Ecol. 2012;21(8):2045-50.

44. Taberlet P, Prud'homme SM, Campione E, Roy J, Miquel C, Shehzad W, Gielly L, Rioux D, Choler P, Clément J-C. Soil sampling and isolation of extracellular DNA from large amount of starting material suitable for metabarcoding studies. Mol Ecol. 2012;21(8):1816-20.

45. Schoch CL, Seifert KA, Huhndorf S, Robert V, Spouge JL, Levesque CA, Chen W, Bolchacova E, Voigt K, Crous PW. Nuclear ribosomal internal transcribed spacer (ITS) region as a universal DNA barcode marker for Fungi. Proc Natl Acad Sci. 2012;109(16):6241-6.

46. Bruns TD, White TJ, Taylor JW. Fungal molecular systematics. Annu Rev Ecol Syst. 1991;22(1):525-64.

47. Coissac E, Riaz T, Puillandre N. Bioinformatic challenges for DNA metabarcoding of plants and animals. Mol Ecol. 2012;21(8):1834-47.

48. Bokulich NA, Mills DA. Improved selection of internal transcribed spacer specific primers enables quantitative, ultra-high-throughput profiling of fungal communities. Appl Environ Microbiol. 2013;79(8):2519-26.

49. Smith DP, Peay KG. Sequence depth, not PCR replication, improves ecological inference from next generation DNA sequencing. PLoS One. 2014;9(2):e90234.

50. Bokulich NA, Thorngate JH, Richardson PM, Mills DA. Microbial biogeography of wine grapes is conditioned by cultivar, vintage, and climate. Proc Natl Acad Sci. 2014:111(1):E139-48.

51. Setati ME, Jacobson D, Bauer FF. Sequence-based analysis of the Vitis vinifera L. cv cabernet sauvignon grape must mycobiome in three south African vineyards employing distinct agronomic systems. Front Microbiol. 2015;6:1358.

52. Bokulich NA, Ohta M, Richardson PM, Mills DA. Monitoring seasonal changes in winery-resident microbiota. PLoS One. 2013;8(6):e66437.

53. Wang C, García-Fernández D, Mas A, Esteve-Zarzoso B. Fungal diversity in grape must and wine fermentation assessed by massive sequencing, quantitative PCR and DGGE. Front Microbiol. 2015:6:1156.

54. Bokulich NA, Collins TS, Masarweh C, Allen G, Heymann H, Ebeler SE, Mills DA. Associations among wine grape microbiome, metabolome, and fermentation behavior suggest microbial contribution to regional wine characteristics. MBio. 2016;7(3):e00631-16.

55. Walters W, Hyde ER, Berg-Lyons D, Ackermann G, Humphrey G, Parada A, Gilbert JA, Jansson JK, Caporaso JG, Fuhrman JA. Improved bacterial $16 \mathrm{~S}$ rRNA gene (V4 and V4-5) and fungal internal transcribed spacer 
marker gene primers for microbial community surveys. Msystems. 2016; 1(1):e00009-15.

56. Busby PE, Peay KG, Newcombe G. Common foliar fungi of Populus trichocarpa modify Melampsora rust disease severity. New Phytol. 2016; 209(4):1681-92.

57. Waring BG, Adams R, Branco S, Powers JS. Scale-dependent variation in nitrogen cycling and soil fungal communities along gradients of forest composition and age in regenerating tropical dry forests. New Phytol. 2016; 209(2):845-54

58. Schloss PD, Westcott SL, Ryabin T, Hall JR, Hartmann M, Hollister EB, Lesniewski RA, Oakley BB, Parks DH, Robinson CJ. Introducing mothur: open-source, platform-independent, community-supported software for describing and comparing microbial communities. Appl Environ Microbiol. 2009;75(23):7537-41.

59. Kashyap PL, Rai P, Kumar S, Chakdar H, Srivastava AK. DNA barcoding for diagnosis and monitoring of fungal plant pathogens. In: Molecular markers in mycology springer; 2017. p. 87-122.

60. Bellemain E, Carlsen T, Brochmann C, Coissac E, Taberlet P, Kauserud H. ITS as an environmental DNA barcode for fungi: an in silico approach reveals potential PCR biases. BMC Microbiol. 2010;10(1):189.

61. Clarke LJ, Soubrier J, Weyrich LS, Cooper A. Environmental metabarcodes for insects: in silico PCR reveals potential for taxonomic bias. Mol Ecol Resour. 2014;14(6):1160-70.

62. Polz MF, Cavanaugh CM. Bias in template-to-product ratios in multitemplate PCR. Appl Environ Microbiol. 1998;64(10):3724-30.

63. Lynch MD, Neufeld JD. Ecology and exploration of the rare biosphere. Nat Rev Microbiol. 2015;13(4):217.

64. Kiss L. Limits of nuclear ribosomal DNA internal transcribed spacer (ITS) sequences as species barcodes for fungi. Proc Natl Acad Sci. 2012;109(27):E1811.

65. Travadon R, Lawrence DP, Rooney-Latham S, Gubler WD, Wilcox WF, Rolshausen PE, Baumgartner K. Cadophora species associated with wooddecay of grapevine in North America. Fungal biol. 2015;119(1):53-66.

66. Úrbez-Torres J, Haag P, Bowen P, O'Gorman D. Grapevine trunk diseases in British Columbia: incidence and characterization of the fungal pathogens associated with esca and petri diseases of grapevine. Plant Dis. 2014;98(4): 469-82

67. Mugnai L, Graniti A, Surico G. Esca (black measles) and brown woodstreaking: two old and elusive diseases of grapevines. Plant Dis. 1999;83(5): 404-18.

68. Larkin MA, Blackshields G, Brown N, Chenna R, McGettigan PA, McWilliam H, Valentin F, Wallace IM, Wilm A, Lopez R. Clustal W and Clustal X version 2.0. bioinformatics. 2007;23(21):2947-8.

69. Walters WA, Caporaso JG, Lauber CL, Berg-Lyons D, Fierer N, Knight R. PrimerProspector: de novo design and taxonomic analysis of barcoded polymerase chain reaction primers. Bioinformatics. 2011;27(8):1159-61.

70. Harris JK, Sahl JW, Castoe TA, Wagner BD, Pollock DD, Spear JR. Comparison of normalization methods for construction of large, multiplex amplicon pools for next-generation sequencing. Appl Environ Microbiol. 2010;76(12): 3863-8.

71. Bengtsson-Palme J, Ryberg M, Hartmann M, Branco S, Wang Z, Godhe A, Wit P, Sánchez-García M, Ebersberger I, Sousa F. Improved software detection and extraction of ITS1 and ITS2 from ribosomal ITS sequences of fungi and other eukaryotes for analysis of environmental sequencing data. Methods Ecol Evol. 2013;4(10):914-9.

72. Edgar RC. Search and clustering orders of magnitude faster than BLAST. Bioinformatics. 2010;26(19):2460-1.

73. Caporaso JG, Kuczynski J, Stombaugh J, Bittinger K, Bushman FD, Costello EK, Fierer N, Pena AG, Goodrich JK, Gordon J. QIIME allows analysis of highthroughput community sequencing data. Nat Methods. 2010;7(5):335.

74. Katoh K, Standley DM. MAFFT multiple sequence alignment software version 7: improvements in performance and usability. Mol Biol Evol. 2013; 30(4):772-80.

75. UNITE_Community. UNITE QIIME release. In: Community U, editor. Version 01.12.2017 edn; 2017.

76. Travadon R, Baumgartner K. Molecular polymorphism and phenotypic diversity in the eutypa dieback pathogen eutypa lata. Phytopathology. 2015; 105(2):255-64.

77. Massonnet M, Morales-Cruz A, Minio A, Figueroa-Balderas R, Lawrence DP, Travadon R, Rolshausen PE, Baumgartner K, Cantu D. Whole-genome resequencing and pan-transcriptome reconstruction highlight the impact of genomic structural variation on secondary metabolite gene clusters in the grapevine esca pathogen Phaeoacremonium minimum. Front Microbiol. 2018;9:1784.

78. Latham SR. Etiology, epidemiology and pathogen biology of esca disease of grapevines in California; 2005.

79. Lawrence DP, Travadon R, Baumgartner K. Diversity of Diaporthe species associated with wood cankers of fruit and nut crops in northern California. Mycologia. 2015;107(5):926-40.

80. Morales-Cruz A, Amrine KC, Blanco-Ulate B, Lawrence DP, Travadon R, Rolshausen PE, Baumgartner K, Cantu D. Distinctive expansion of gene families associated with plant cell wall degradation, secondary metabolism, and nutrient uptake in the genomes of grapevine trunk pathogens. BMC Genomics. 2015;16(1):469.

81. Massonnet M, Morales-Cruz A, Figueroa-Balderas R, Lawrence DP, Baumgartner K, Cantu D. Condition-dependent co-regulation of genomic clusters of virulence factors in the grapevine trunk pathogen Neofusicoccum parvum. Mol Plant Pathol. 2018;19(1):21-34.

82. Bolger AM, Lohse M, Usadel B. Trimmomatic: a flexible trimmer for Illumina sequence data. Bioinformatics. 2014;30(15):2114-20.

\section{Ready to submit your research? Choose BMC and benefit from:}

- fast, convenient online submission

- thorough peer review by experienced researchers in your field

- rapid publication on acceptance

- support for research data, including large and complex data types

- gold Open Access which fosters wider collaboration and increased citations

- maximum visibility for your research: over $100 \mathrm{M}$ website views per year

At BMC, research is always in progress.

Learn more biomedcentral.com/submissions 